\title{
REVISÃO BIBLIOGRÁFICA: DOSES DE RADIAÇÃO IONIZANTE NO CONTROLE DA PODRIDÃO EM MORANGOS PÓS-COLHEITA
}

\author{
Estefanie C. de Farias* \\ Departamento de Física - UTFPR - PR \\ *Autor correspondente, email: estefaniefarias@gmail.com
}

\begin{abstract}
Resumo - Foi realizado uma revisão de literatura sobre a utilização de radiação ionizante no controle da podridão, como forma de conservação de morangos em sua fase de pós-colheita. Neste artigo foi abordado alguns tipos de radiação para que seja feito esse controle, como radiação gama e sua utilização na conservação de alimentos. $O$ artigo mostra vários estudos que foram investigados e os resultados destes estudos, no qual demonstram a eficiência da irradiação com cobalto-60 e também da quantidade de dose na eliminação de microorganismos que afetam a pós-colheita do morango, fazendo com que se tenha maior índice de podridão e maior desperdício do produto. A revisão de literatura foi feita e como resultados finais observou que doses abaixo de $1 \mathrm{kGy}$ foram irrelevantes para o aumento da vida útil dos morangos. Já doses entre 1,5 e 3,0 kGy obtiveram ótimos resultados.
\end{abstract}

Palavras-chave: irradiação de morangos; conservação de alimentos; radiologia industrial; revisão de literatura.

\section{Introdução}

O morango é um pseudofruto muito apreciado devido a sua aparência atraente e sabor característico, seu consumo produz benefícios à saúde humana com efeitos antioxidante, anti-inflamatório e anticarcinogênico, atuando na melhoria da qualidade de vida dos indivíduos [1]. Em decorrência da alta produtividade e do seu gosto atrativo, o morango talvez seja considerado o fruto de maior importância entre as frutas pequenas. Apesar das dificuldades e dos riscos que envolvem a sua produção, o cultivo do morango é muito lucrativo [2]. Entretanto o fruto é de alta perecibilidade, tendo validade de 2-3 dias à temperatura ambiente e é vulnerável à manipulação pós-colheita por causa de sua taxa de respiração, estresses de transporte e ataques patogênicos [3]. Cerca de 30-40\% dos frutos são perdidos entre a colheita e consumo devido à sua fragilidade [4]. Sendo assim, tecnologias de conservação que evitem ao máximo essas alterações, as quais são, na maioria das vezes, indesejáveis, devem ser utilizadas no seu beneficiamento [5,6]. Uma das tecnologias que pode ser aplicada é a refrigeração, em que a baixa temperatura reduz o metabolismo de frutos durante a pós-colheita e é um importante fator na manutenção da qualidade dos mesmos [7]. Atmosfera controlada no pacote $\left(\mathrm{CO}^{2}, \mathrm{O}^{2}\right)$ e baixa temperatura no armazenamento são técnicas convencionais e populares para o controle de maturação e extensão da vida útil de frutas e hortaliças [8]; entretanto, em vários estudos foi documentado que esses métodos já não são mais suficientes para conter os fungos e bactérias patogênicos que estão nas condições de armazenamento [9,10]. Com isso a radiação ionizante vem sendo aplicada com diversos propósitos. Destaca-se a sua aplicação em alimentos, área em que se tornou promissora técnica de conservação em combinação ou substituição ao tratamento térmico. Quando doses controladas são administradas, as alterações nas características nutricionais e sensoriais do alimento são mínimas. Além disso, pode ser aplicada em alimentos sólidos e líquidos já embalados, evitando a recontaminação pós tratamento $[11,12]$. Esta revisão de literatura tem como objetivo abordar pesquisas relacionadas e que foram realizadas a irradiação com variação de dose em morangos. São apresentados resultados e discussões de avaliações que autores diversos fizeram com o intuito de observar o limiar de dose necessário para controlar os microrganismos e visando manter o produto com uma maior longevidade.

Revisão Bibliográfica

O morango

O morango é um pseudofruto amplamente produzido e consumido no mundo inteiro. O fruto no entanto é altamente perecível, com tempo de vida-de-prateleira de 2-3 dias à temperatura ambiente, sendo extremamente vulnerável à pós-colheita por causa de sua alta taxa de respiração, estresse ambiental e ataques patogênicos [3]. No Brasil a área cultivada com o morangueiro é de aproximadamente 4300 hectares, estimando-se uma produção anual de 155 mil 
toneladas. O fruto possui ampla adaptabilidade às mais diversas condições edafoclimáticas, assim seu cultivo é possível em países de clima temperado a tropical [13].

\section{Controle de microrganismos}

As frutas naturais apresentam grande quantidade de microrganismos por isso é preciso que estas passem por algum processamento para a eliminação dos mesmos, eliminando assim a probabilidade de contaminações alimentares. Dessa forma é exigido que o processo proporcione a segurança microbiana do produto, preservando as características sensoriais e nutricionais semelhante à matéria-prima [14]. A irradiação dos alimentos é um método utilizado como pré-processamento de produtos resfriados, uma vez que tem a capacidade de destruir microrganismos patogênicos e deteriorantes presentes nos alimentos [15,16]. São recomendadas doses de até $1 \mathrm{kGy}$ para inibir infestação de insetos e amadurecimento tardio, entre 1 e $10 \mathrm{kGy}$ para reduzir a carga bacteriana, entre eles os microrganismos patogênicos. Para esterilização comercial e eliminação de vírus são indicadas doses entre 10 e 50 kGy [17,18]. A eficácia da irradiação gama em diminuir o deterioração de frutas e vegetais é associada com sua habilidade de penetração profunda no tecido e destruição dos microrganismos encontrados ao redor ou dentro dos tecidos, prevenindo ou diminuindo o processo de apodrecimento pela inibição do crescimento de microrganismos [19].

\section{A irradiação gama com cobalto 60}

A aplicação da radiação gama têm gerado resultados satisfatórios em diferentes aspectos da tecnologia de alimentos, como esterilizações, inibição de esporos e aumento da vida útil de frutas e hortaliças [20]. Existem duas fontes de radiação, o Cobalto-60 (60-Co) e o Césio-137 (137-Cs), considerados para uso comercial, devido à emissão de raios gama de energias adequadas. Sendo o 60-Co o mais utilizado devido à sua forma metálica e à insolubilidade em água, gerando maior segurança ambiental $[21,22,23]$. A irradiação de alimentos é aprovada por órgãos como o Food and Drug Administration, o Codex Alimentarius Commission, a World Health Organization, a American Medical Association e o Institute of Food Technologies [24,25]. Segundo Kume [26] mais de 60 países possuem legislação aprovando o uso da tecnologia em torno de 100 tipos de alimentos. O processo consiste na exposição dos alimentos, embalados ou a granel, a uma dose controlada de radiação ionizante em um determinado período de tempo [27,28]. Atualmente a ANVISA regulamenta as normas para o uso da irradiação para alimentos no Brasil com a RDC n ${ }^{\circ} 21$ de 26 de janeiro de 2001 a qual informa que qualquer alimento poderá ser tratado por radiação desde que a dose mínima absorvida seja o suficiente para alcançar a finalidade pretendida e a dose máxima absorvida seja inferior àquela que comprometeria a as propriedades funcionais e ou os atributos sensoriais do alimento. O rótulo dos alimentos irradiados deve possuir o símbolo internacional da radiação ionizante, chamado Radura (Fig.1) [29].

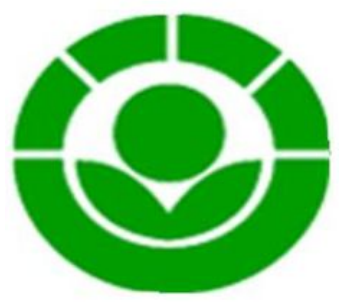

Fig.1 - Radura, símbolo internacional que identifica alimentos irradiados [29].

A instalação industrial de irradiação de alimentos deve ser licenciada, regulamentada e inspecionada pelas autoridades nacionais de segurança radiológica e de saúde. A sala de irradiação precisa possuir blindagem de concreto para garantir que a radiação ionizante não escape para fora da sala [30,31].

\section{Discussão}

Com o objetivo de avaliar a variação do tempo de validade com relação à dose aplicada nos morangos, alguns estudos recentes como o de Wani et al. [32] demonstrou que, doses entre 1.5 kGy e 1,7 kGy de radiação são responsáveis por manter a qualidade das frutas em até 14 dias de armazenamento.

No trabalho de Mridha et al. [33] feito em 2017, os autores obtiveram resultados parecidos avaliando-se os efeitos das doses de irradiação gama $(0 ; 0,5 ; 1,0$ e $1,5 \mathrm{kGy})$ em morangos durante 6 dias de armazenamento. O estudo mostrou que o morango não irradiado estragou completamente dentro dos 6 dias, enquanto que as amostras que receberam radiação gama de 1,5 kGy permaneceram adequadas para consumo com base nas análises microbiológicas. Os autores notaram que durante o armazenamento houve um aumento de bactérias e fungos tanto para os morangos não irradiados quanto para os irradiados na dose mais baixa, dessa forma foi percebido que doses baixas não eliminam todos os microrganismos, mas reduzem a contaminação total. 
Prof. Dr. Walmor Cardoso Godoi

Com essas mesmas doses o trabalho de Nassur [7] em 2016, com um sistema refrigerado $\left(0 \pm 1^{\circ} \mathrm{C}\right.$ e $90 \pm 5 \%$ UR $)$ por 15 dias. E encontrou que a irradiação gama nas doses de 1,0 KGy e 1,5 KGy mantiveram menor perda de massa fresca e maior atividade antioxidante logo após a aplicação dos tratamentos, prolongando a vida útil de morangos com qualidade. Também neste estudo a autora concluiu que a irradiação gama na dose de 1,5 KGy controla visualmente a podridão dos morangos.

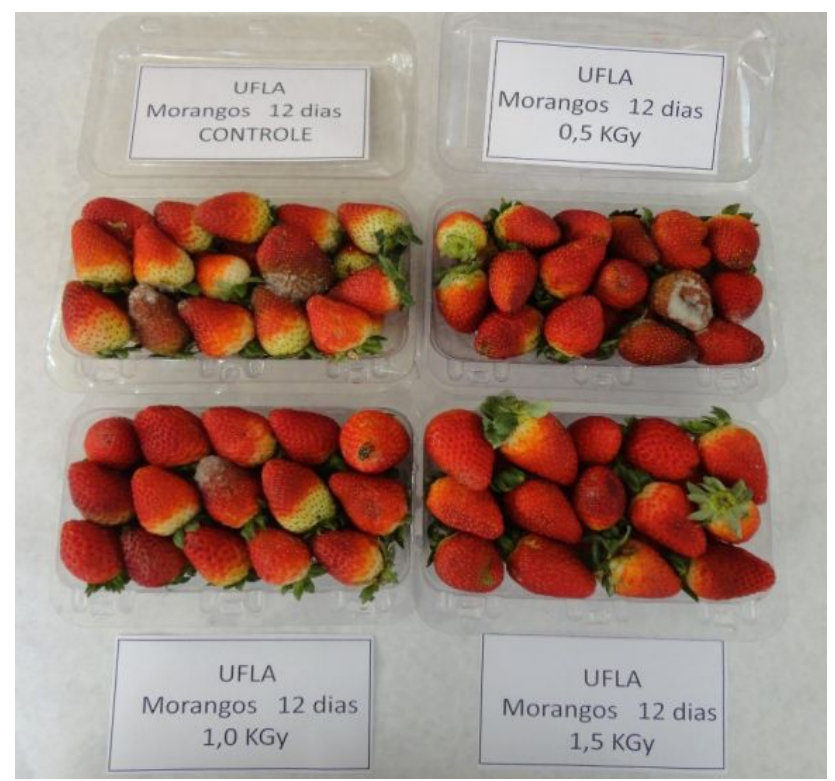

Fig.2 - Morangos submetidos a doses de irradiação gama (0; 0,5; 1,0; 1,5 KGy) e armazenados por 12 dias a $4 \pm 1$ oC e $90 \pm 5 \%$ UR.

Uma outra pesquisa feita recentemente no Brasil por Quintão [34] com a utilização de quatro níveis do fator irradiação ( $0 ; 0,8 ; 1,6$ e 2,4 kGy) e quatro níveis do fator tempo de armazenamento (0,3, 6 e 9 dias). A irradiação em baixas doses e sob refrigeração possibilitou um acréscimo de três dias no período de comercialização dos morangos. O estudo mostrou também que as amostras não irradiadas tornaram-se impróprias para consumo depois do sexto dia de armazenamento, enquanto as amostras que receberam radiação gama mantiveram as qualidades físico-químicas e microbiológicas ao longo dos nove dias de armazenamento. Os frutos irradiados na dose 2,4 kGy mostraram-se mais eficiente no controle dos microrganismos durante o período avaliado (Tab.1), sendo esta a dose mais ideal a ser utilizada para o aumentar a vida útil dos morangos.

\begin{tabular}{|c|c|c|c|}
\hline $\begin{array}{l}\text { Tempo } \\
\text { (dias) }\end{array}$ & $\begin{array}{c}\text { Doses } \\
\text { (kGy) }\end{array}$ & $\begin{array}{l}\text { Coliformes Totais } \\
\text { (NMP } \mathrm{g}^{-1} \text { ) }\end{array}$ & $\begin{array}{c}\text { Bolores e leveduras } \\
\text { (UFC } \mathrm{g}^{-1} \text { ) }\end{array}$ \\
\hline \multirow[t]{4}{*}{0} & 0 & $<3,0$ & $6,55 \times 10^{5}$ \\
\hline & 0,8 & $<3,0$ & $5,90 \times 10^{5}$ \\
\hline & 1,6 & $<3,0$ & $7,35 \times 10^{3}$ \\
\hline & 2,4 & $<3,0$ & $7,75 \times 10^{3}$ \\
\hline \multirow[t]{4}{*}{3} & 0 & 1100 & $1,46 \times 10^{6}$ \\
\hline & 0,8 & 150 & $>5,00 \times 10^{5}$ \\
\hline & 1,6 & 15 & $7,50 \times 10^{5}$ \\
\hline & 2,4 & 3,6 & $>5,00 \times 10^{5}$ \\
\hline \multirow[t]{4}{*}{6} & 0 & 1100 & $5,00 \times 10^{5}$ \\
\hline & 0,8 & 460 & $9,65 \times 10^{5}$ \\
\hline & 1,6 & 43 & $1,94 \times 10^{6}$ \\
\hline & 2,4 & 9,2 & $4,30 \times 10^{5}$ \\
\hline \multirow[t]{4}{*}{9} & 0 & * & * \\
\hline & 0,8 & 1100 & $5,05 \times 10^{5}$ \\
\hline & 1,6 & 12 & $5,50 \times 10^{4}$ \\
\hline & 2,4 & 23 & $2,64 \times 10^{4}$ \\
\hline
\end{tabular}

Tab.1 - Resultado das análises microbiológicas para morangos tratados com diferentes doses de irradiação gama $(0 ; 0,8 ; 1,6 ; 2,4 \mathrm{kGy})$ armazenados por até 9 dias sob refrigeração $\left(10 \pm 1^{\circ} \mathrm{C}\right) \mathrm{e} 90 \pm 5 \% \mathrm{UR}$ [34].

No trabalho do paquistanês Majeed [35] em 2014, utilizou as doses de 0,5; 1,0 e 1,5 kGy com um armazenamento de nove dias à temperatura ambiente. Em seus resultados observou que houve um aumento de vida de 5,75 e 7,75 dias nas amostras irradiadas com 1,0 e 1,5 kGy respectivamente quando comparado ao controle e 0,5 kGy onde o tempo de vida no armazenamento do fruto teste foi praticamente 3 dias (Fig.3). Na amostra de controle, após esses três dias, a deterioração dos morangos aumentou gradativamente, fazendo deles inaceitáveis. Resultados similares foram obtidos com $0,5 \mathrm{kGy}$, mesmo que esta dose tenha aumentado bem pouco a vida útil mas o aumento não foi significativo. $\mathrm{O}$ autor então observou que com os resultados obtidos o trabalho sugere que irradiação gama de mais de 1,5 kGy pode ser eficiente na diminuição da deterioração, aumentando a vida útil do fruto sem causar mudanças em sua composição. 


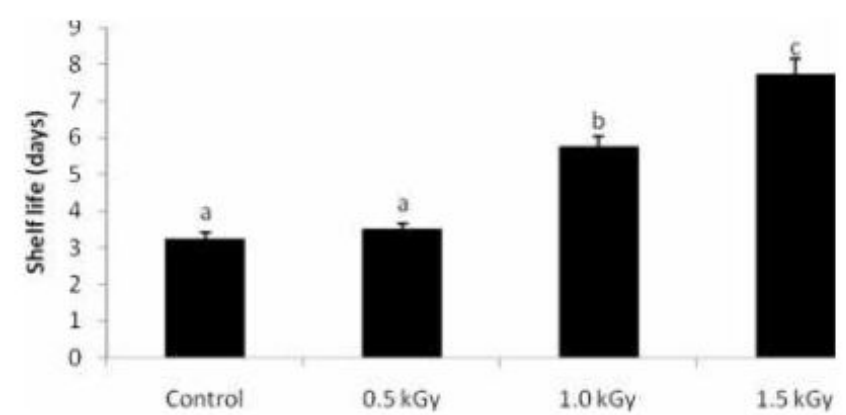

Fig. 3 -Efeito da irradiação gama na vida útil de morangos armazenados à temperatura ambiente [35].

$\mathrm{Na}$ pesquisa de Silva et al. [36] foram utilizadas doses maiores (2,0 e 2,5 kGy) e tempo de armazenamento de duas semanas à temperatura de $12,5^{\circ} \mathrm{C}$. Com sete dias de armazenamento, a amostra de controle já tinha mais da metade em processo de decomposição; e após nove dias, elas foram descartadas. As frutas irradiadas, no entanto, ainda não tinham nenhum sinal de deterioração. Os autores observaram também que no teste de aceitação as doses de 2,0 e $2,5 \mathrm{kGy}$ não obtiveram resultados diferentes. E depois das duas semanas, a observação da parte externa dos frutos, houve $67 \%$ de aceitação das frutas que receberam a dose de $2,5 \mathrm{kGy}$ e $71 \%$ para aquelas que receberam $2,0 \mathrm{kGy}$.

O trabalho de Filho [37] utilizou doses maiores, até 3,6 kGy e obteve resultados melhores do que as pesquisas com até 2,5 kGy. Foram realizadas análises microbiológicas de fungos e leveduras em morango controle e irradiado (nas doses de $0,405 \mathrm{kGy}$ e $3,6 \mathrm{kGy}$ ) armazenados a $8 \mathrm{C}$, nos dias 1,8 e 15 de armazenamento. E a análise microbiológica demonstrou que a irradiação do morango na dose 3,6 kGy foi efetiva na diminuição da carga microbiana de todos os microrganismos em estudo, já a dose de $0,405 \mathrm{kGy}$ não apresentou a mesma eficiência. E neste estudo também, o autor verificou que dos microrganismos analisados, os fungos são os mais resistentes à radiação. Por meio de análises microbiológicas constatou-se que a dose de 3,6 kGy é efetiva na diminuição da carga microbiana de fungos e consequentemente da podridão em até 15 dias de armazenamento.

De acordo com Thomas [38], para estender a vida de prateleira de morangos é recomendada a utilização de doses de radiação de 1,5 kGy a 3,0 kGy. Doses abaixo não atenderem com eficiência à finalidade pretendida e doses acima resultam em textura externa mais macia e perda da cor vermelha, algo que não foi verificado na pesquisa acima com doses de 3,6 kGy.

Por fim, um trabalho de compilação da International Atomic Energy Agency (IAEA), em 1994 [39] publicou este trabalho com recomendações da irradiação em morangos. Em que recomenda doses maiores do que 3,0 kGy de irradiação gama associado com armazenamento em temperatura baixa $\left(-1,5^{\circ} \mathrm{C}\right)$ para estender a vida útil e diminuir a incidência de fungos e leveduras em morangos.

\section{Considerações Finais}

A irradiação de alimentos, neste caso o morango, em que a radiação ionizante do tipo gama agiu na conservação de alimentos, com o intuito de inativar microrganismos patogênicos, retardar o amadurecimento, desinfetar o morango. De acordo com as pesquisas aqui mostradas, o morango sofreu uma grande variação de validade dependendo da dose aplicada à ele em comparação à amostra de controle e as minimamente irradiadas ( 0 kGy - 0,5 kGy). As irradiações de doses mais altas (1,5 kGy - 3,6 kGy) melhoraram visivelmente o tempo de vida útil dos morangos nos estudos apresentados aqui. Sabe-se que o processo de irradiação de alimentos é seguro e certificado no Brasil, sendo uma alternativa barata e de longa-vida, mesmo com um custo inicial elevado. Por fim, um estudo mais aprofundado é necessário para se observar quais as doses e por quanto tempo pode-se armazenar as amostras em temperatura ambiente e/ou na refrigeração, para que o produto dure mais e também mantenha suas características físico-químicas para ser consumido.

\section{Agradecimentos}

Gostaria de agradecer ao professor Walmor C. Godoi, em que se encontra na regência da matéria de Radiologia Industrial, na qual este artigo foi idealizado e feito. Gostaria também de agradecer à instituição UTFPR pela oportunidade e por me indagar cada vez mais à seguir um caminho acadêmico.

\section{Referências}

[1] Da Silva Pinto, M., Lajolo, F.M., Genovese, M.I.2008. Bioactive compounds and quantification of total ellagic acid in strawberries (Fragaria ananassa Duch.). Food Chemistry 107:1629-1635. 
[2] M. I. Padovani. Morango: o delicado e saboroso fruto da integração dos povos. São Paulo: Ícone, 1991. 68p. (Coleção Brasil Agrícola).

[3] M. Zhang, G. Xiao, J. Peng, V.M. Salokhe. Effect of modified atmosphere package on preservation of strawberries. International Agrophysics, 17 (3) (2003), pp. 143-148.

[4] M. Yarahmadi; M. Azizi; B. Morid; S. Kalatejari. 2014. Postharvest application of gum and mucilage as edible coating on postharvest life and quality of strawberry fruit. International Journal of Advanced Biological and Biomedical Research.

[5] I.L.T. Françoso; M.A.L. Couto, M.A.L; S. G. Canniati-Brazaca; Arthur, V. 2008. Alterações físico-químicas em morangos (Fragaria anassa Duch.) irradiados e armazenados. Ciência e Tecnologia de Alimentos.

[6] A. R. Ponce; D.I.M. Bastiani; P.V. Minim; D.C.M. Vanetti. Características físico-químicas e microbiológicas de morango minimamente processado. Ciência e Tecnologia de Alimentos. 2010, vol.30, n.1, pp.113-118.

[7] R. de C. M. R. Nassur; R. A. Z. Lima; L. C. O. Lima; N. N. J. Chafun. Doses de radiação gama na conservação da qualidade de morangos. Comunicata Scientiae, Bom Jesus, v. 7, n. 1, p. 38-48, 2016.

[8] R.C. Wiley. Minimally processed refrigerated fruits and vegetables. Chapman and Hall, New York. pp. 368. 1994.

[9] M. E. Berrang; R.E. Brackett; L.R. Beuchat. Growth of Listeria monocytogenes fresh vegetables stored under controlled atmosphere. J. Food Prot. 1989.

[10] S.S. Sumner; D. L. Peters. Microbiology of vegetables. In: Smith, D. S., J. N. Cash, W. K.Nip and Y. H. Hui (eds.). Processing vegetables science and technology.Technomic publishing Co, Inc. Lancaster, Pa. pp.87-106. 1997.

[11] N.K. Hernandes ; H. C.Vital ; A. U. O. Sabaa Srur. Irradiação de alimentos. Boletim SBCTA, v. 37, n. 2, p. 154-159, 2003.

[12] P.J. Fellows. Tecnologia do processamento de alimentos:princípios e prática. 2.ed. Porto Alegre: Artmed, 2006. $602 \mathrm{p}$.

[13] H. Stockhausen; B.F. De Oliveira; K.V.T. Dos Santos; R. Martins; M.F.S. Dos Santos; A.F. Fagherazzi; D.S. Zanin; R. Rufato. Adaptabilidade de genótipos de morangueiro no planalto catarinense. Revista Uniplac, v.6, n.1, 2018.

[14] H. Song; M. Byun; C. Jo; C. Lee; K. Kim; D. Kim. Effects of gamma irradiation on the microbiological, nutritional, and sensory properties of fresh vegetable juice. Food Control, v.18, p.5-10, 2007.

[15] C.B.D. Ornellas; M.P.J. Gonçalves; P.R. Silva; R. T. Martins, Atitude do consumidor frente à irradiação de alimentos. Ciência e Tecnologia de Alimentos, v.26, n.1, p.211-213, 2006.

[16] M.C. Souza; L.J.Q. Teixeira; C.T. Rocha; G.A.M. Ferreira; T.L. Filho. Emprego do frio na conservação de alimentos. Enciclopédia Biosfera, v.9, n.16; p. 1027-1046, 2013.

[17] M. Jouki; N. Khazaei. Effect of low-dose gamma radiation and active equilibrium modified atmosphere packaging on shelf life extension of fresh strawberry fruits. Food Packaging and Shelf Life, 1, 49-55. 2014.

[18] WHO. World Health Organization.High dose irradiation: wholesomeness of food irradiated with doses above 10 kGy. Geneva, 1999. 204 p. (Technical Report Series, 890).

[19] R. Barkai-Golan. Postharvest Diseases of Fruits and Vegetables: Development and Control. Elsevier Sci. Press. 2001. 
[20] M.I. Egea; M.C. Sanchez-Bel; F.B. Martinez Madrid; F.B. Flores; F. Romojaro. The effect of beta ionization on the antioxidant potential of "Bulida" apricot and its relationship with quality. Postharvest Biology and Technology 46: 63-70. 2007.

[21] A.J. Campos. Radiação gama, ultravioleta (uv-c) e atmosfera controlada na conservação da qualidade de tomate 'débora plus'e 'pitenza'. 2008. 138 f. Dissertação (Doutorado em Agronomia) -Universidade Estadual Paulista Julio de Mesquita Filho, Botucatu, São Paulo, 2008.

[22-23] A.L.F. Silva; C.R. Roza. Uso da irradiação em alimentos: revisão. Boletim CEPPA, Curitiba, v.28, p.49-56, 2010 .

[24] K. Akram; J.H.Kwon. Food Irradiation for Mushrooms: A Review J. Korean Soc. Appl. Biol. Chem. 53(3), 257-265 doi:10.3839/jksabc.2010.041. 2010.

[26] T. Kume; M. Furata; S. Todoriki; N. Uenoyama; Y. Kobayashi. Quantity and Economic Scale of Food Irradiation in the World. RADIOISOTOPES. 58. 25-35. 10.3769/radioisotopes.58.25. 2009.

[27] A.C.L. Castelucci. Avaliação da estabilidade dos compostos bioativos de polpas de frutas nativas submetidas ao processo de irradiação. 2015. 133 f. Tese (Doutorado em Ciências) -Centro de Energia Nuclear na Agricultura, Universidade de São Paulo, Piracicaba, 2015.

[28] CENTRO DE ENERGIA NUCLEAR NA AGRICULTURA E NO MEIO AMBIENTE (CENA). Divulgação da tecnologia da irradiação de alimentos e outros materiais. 2015.

[29] BRASIL. Agência Nacional de Vigilância Sanitária (ANVISA). Resolução n²1, de 26 de janeiro de 2001. Aprova regulamento técnico para irradiação de alimentos. Diário Oficial União, Brasília, DF, 2001.

[30] C. A. Fante; Caracterização, Qualidade e Conservação Pós-Colheita de maçã 'Eva' (Malus sp.). Dissertação (Pós-Graduação em Ciência de Tecnologia de Alimentos) - Universidade Federal de Minas Gerais, Belo Horizonte, 2011.

[31] Centro de Desenvolvimento da Tecnologia Nuclear (CDTN/UFMG). Fatos sobre irradiação de alimentos. Belo Horizonte: CDTN, 1999. 46 p.

[32] A. M. Wani; P.R. Hussain; R.S. Meena; M.A. DAR. Effect of gamma irradiation and refrigerated storage on the improvement of quality and shelf-life of pear (Pyrus Communis L., Cv.Bartlett/William). Radiation physics and chemistry, v.77, p. $983-989,2008$.

[33] F. Mridha; Roksana H.; M.S.T. Khatum; M. Islam; Mst; A. Hossain. Effects of gamma irradiation on antioxidant markers, microbial population and sensory attributes of strawberry (Fragaria $\times$ ananassa Duch.) cv. Festival. 2017.

[34] A.L Quintão; Efeitos da Radiação Gama na Qualidade de Morangos Scv. ALBION. Dissertação (Pós-Graduação em Ciência de Tecnologia de Alimentos) - Universidade Federal de Minas Gerais, Belo Horizonte, 2018.

[35] A. Majeed; Z.Muhammad; A. Majid; A.H. Shahand; M. Hussain; Impact of Low Doses of Gamma Irradiation on Shel Life and Chemical Quality of Strawberry (Fragaria Ananassa) CV. 'CORONA'. The Journal of Animal \& Plant Sciences, 24(5): 2014, Page:1531-1536ISSN: 1018-7081

[36] M.A. Silva; S. Aquino; R.L. Guedes; P.V. Silva; R.A. Trindade; M.P. Pedroso; A.L.C.H. Villavicencio. Avaliação sensorial de mandioca pré-cozida submetida a diferentes doses de radiação. Arq. Inst. Biol., São Paulo, v. 72, supl.2, p. $1-64,2005$. 
RADIOLOGIA INDUSTRIAL - 2019-1

Prof. Dr. Walmor Cardoso Godoi

[37] T. Lima Filho. Strawberry irradiation: determination of detection threshold and consumer rejection threshold and the impact of preservation technology on consumers acceptance and perceptions. 2013. $227 \mathrm{f}$. Dissertação (Mestrado em Ciência de Tecnologia de Alimentos) - Universidade Federal do Espírito Santo, Alegre, 2013.

[38] P. Thomas. Irradiation of strawberries: a compilation of technical data for its authorization and control. In: MEETING OF THE INTERNATIONAL CONSULTATIVE GROUP OF FOOD IRRADIATION (ICGFI), 10. Geneva: WHO, 1993. 37p.

[39] IAEA. Irradiation of strawberries. A compilation of technical data for its authorization and control. FAO/IAEA/WHO IAEA-TECDOC.pp.779:34. 1994. 